\title{
Heparinized and Saline Solutions in the Maintenance of Arterial and Central Venous Catheters After Cardiac Surgery
}

\author{
Mohsen Ziyaeifard ${ }^{1}$; Azin Alizadehasl ${ }^{1}$; Nahid Aghdaii ${ }^{1,}$; Ali Sadeghi ${ }^{1}$; Rasoul Azarfarin ${ }^{1}$; \\ Gholamreza Masoumi ${ }^{2}$; Ghodrat Golbargian ${ }^{1}$ \\ ${ }_{1}^{1}$ Rajaie Cardiovascular Medical and Research Center, Iran University of Medical Sciences, Tehran, Iran \\ ${ }^{2}$ Department of Anesthesiology, Isfahan University of Medical Sciences, Isfahan, Iran \\ ${ }^{*}$ Corresponding author: Nahid Aghdaii, Rajaie Cardiovascular Medical and Research Center, Iran University of Medical Sciences, Tehran, Iran. Tel: +98-2123922148 Fax: +98-22663293, \\ E-mail:Aghdaii @yahoo.com
}

Received: February 17, 2015; Revised: April 11, 2015; Accepted: May 2, 2015

\begin{abstract}
Background: Heparinized saline solution is used to prevent occlusion in the arterial catheters and central venous pressure monitoring catheters. Even at low dose, heparin administration can be associated with serious complications. Normal saline solution can maintain patency of arterial catheters and central venous pressure monitoring catheters.

Objectives: The current study aimed to compare the efficacy of normal saline with that of heparinized one to maintain patency of arterial and central venous catheters after cardiac surgery.

Patients and Methods: In the current randomized controlled trial, 100 patients, with an age range of 18 - 65 years of valve and coronary artery surgery were studied in Rajaie heart center, Tehran, Iran. Patients were randomized to receive either heparinized saline $(n=50)$ or normal saline flush solutions $(n=50)$. In the study, arterial catheters and central venous pressure monitoring catheters were daily checked for any signs of occlusion in three postoperative days as primary end-point of the study.

Results: According to the information obtained from the study, four (8\%) arterial catheters in the saline group (P value: 0.135) and three $(6 \%)$ arterial catheters in the heparin group (Pvalue $=0.097$ ) were obstructed. Statistical analysis showed that the incidence of obstruction and changes in all other parameters between the two groups during the three-day follow-up was not significant (all P values $>0.05$ ).

Conclusions: It seems that there is no difference in the use of heparinized and normal saline solutions to prevent catheter occlusion of arterial and central venous pressure.
\end{abstract}

Keywords: Catheterization; Saline Solution; Heparin; Cardiac Surgery

\section{Background}

To care for patients who need to access to the central venous system, central venous catheters, including internal and external jugular vein, subclavian vein, axillary vein and femoral vein are used to administer fluids, medications, parenteral nutrition, central venous pressure monitoring, blood products and blood sampling $(1,2)$. Arterial catheters include radial, brachial, femoral, dorsalis pedis and axillary in the intensive care unit are used for blood pressure monitoring, repeated blood sampling, and the inability to measure indirect blood pressure (3) ; invasive arterial blood pressure measurements are more accurate (4).

Heparinized solution is used to prevent occlusion in these catheters. Heparin is an anticoagulant drug used to prevent and treat thrombosis (5). Unfractionated heparin is a standard anticoagulant that affects multiple sites of internal and external coagulatory system and inhibits blood clotting $(6,7)$. Heparin, even with low dose, can cause thrombocytopenia and bleeding (8-10). The absolute risk of heparin-induced thrombocytopenia (HIT) with unfractionated heparin is $1 \%-5 \%(11,12)$.
Normal or isotonic saline has sodium and chloride concentrations of $154 \mathrm{mEq} / \mathrm{L}$ (13). Normal saline solution can maintain patency of arterial and central venous pressure monitoring catheters. Heparinized solutions had no effect on prolonging patency and improving function of catheters and even caused changes in activated partial thromboplastin time (APTT). While the normal saline solution increased accuracy of coagulatory tests. Use of normal saline solution prevents patient exposure to the risks associated with heparinized solution that increase patient safety $(5,14,15)$.

\section{Objectives}

The current study aimed to compare the efficacy of normal and heparinized saline to maintain patency of arterial and central venous catheters after cardiac surgery.

\section{Patients and Methods}

The current study was a double blind randomized 
clinical trial. The study proposal was approved in our institutional ethics committee and all patients singed informed written consent before entering the study. Only single valve and isolated oronary artery bypass graft (CABG) surgeries were studied. Usually in valve patients, warfarin changed to heparin several days before surgery and heparin was dis-continued on the day of operation. In this randomized controlled trial, 100 patients of 18 - 65 years, undergone valve and coronary artery surgery were studied in Rajaie heart center, Tehran, Iran. Patients were randomized to receive either heparinized $(n=50)$ or normal saline flush solutions $(n=50)$. The patients were randomly allocated in two groups by online randomizer software (http://www.graphpad.com/ quickcalcs/randomize2/). Considering the frequencies of catheter occlusion the difference between heparinized and normal saline solutions, $8 \%$ and $18 \%$ in Kulkarni et al. (5) study and $\alpha=0.05$ and $\beta=0.1$, the calculated number of patients in each group was 35 . The researcher increased sample size to 50 to overcome some degrees of loss to follow up. The inclusion criteria were: arterial and central venous catheters, Prothrombin time $(\mathrm{PT})<13$ seconds, partial thromboplastin time $(\mathrm{PTT})<$
45 seconds, platelet count $140000-440000 \mathrm{~mm}^{3}$, older than 18 and younger than 65, filling out a consent form for the study and placement of catheters in intensive care unit. The exclusion criteria were: known allergy to heparin, platelet treatment, history of heparin-induced thrombocytopenia, coagulation disorder before using heparin, more than five times effort to put the catheter in place, thrombocytopenia with platelets less than $100,000 \mathrm{~mm}^{3}$ and simultaneous participation in other studies. Study was frequency of arterial/venous catheter patency in three postoperative days.

\subsection{Materials and Drugs}

Arterial /venous measurement transducer was used (Edwards Lifescience, Dominican Republic). The transducers were calibrated, zeroed and leveled at the beginning of each working shift and also when the measurement faced any problems. Heparin 5,000 units (ABURAIHAN Co., Tehran, Iran) and normal saline solution $500 \mathrm{~mL}$ (SAMEN Co., Mashhad, Iran) were used. Data were gathered daily for any signs of occlusion. Patient follow up was conducted only for three postoperative days in intensive care unit (ICU). There was no follow up loss (Figure 1).

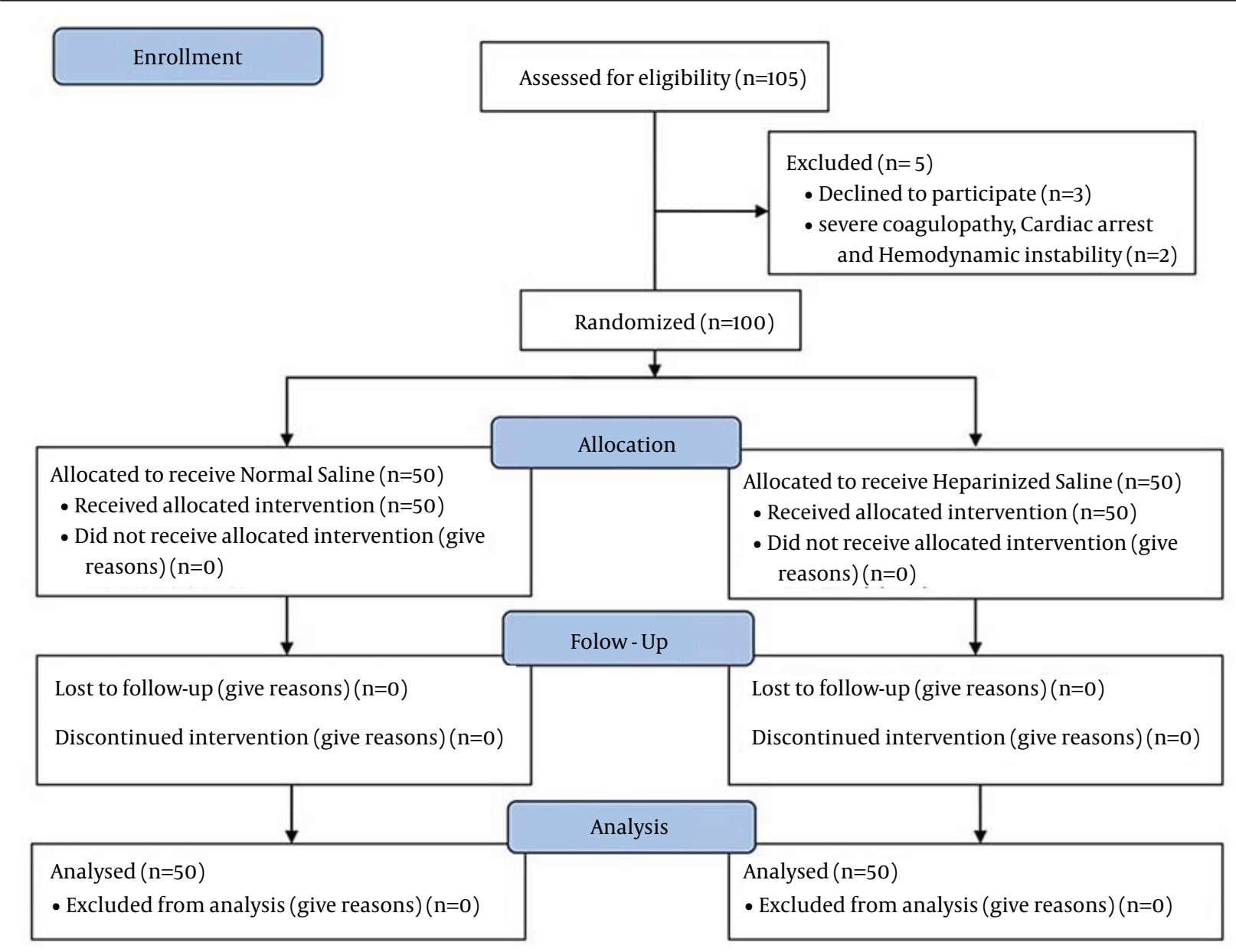

Figure 1. The Study Flow Diagram 
Only hemodynamically stable patients were studied, and mostly radial artery was used for catheterization (gauge 20) and internal right jugular vein CV line placement (three lumen catheters from one manufacture (B BRAUN), with lumen sizes 16, 20, 20 gauge); therefore it was tried to minimize probable confounding effects of catheter size and placement site. Catheterization technique was the same in all patients of both groups. Catheters were examined based on the checklist from the items used in previous studies (16). Check for occlusion in the artery catheters based-on blood return and monitoring devices were connected to the patient. If blood returned, catheter was opened. Arterial catheter should be an appropriate response to the flush test. It means that with the serum flush in the arterial catheter, arterial wave disappeared and viewed direct line in the monitoring device. Sing of the catheter should also have a rapid decline to below the baseline. The flash test was used to check the venous catheter occlusion blood return to the catheter and positive response. In the control and intervention groups, to maintain patency of arterial and central venous catheters normal and heparinized saline solutions with concentration $10 \mathrm{u} / \mathrm{mL}$ were used, respectively. Among the patients if the central venous catheters were used continuously, there was no need for any flush solution, but if used intermittently, in the control group first it was washed with 10 $\mathrm{mL}$ of normal saline solution then locked with $5 \mathrm{~mL}$ of the same saline solution and in the intervention group, central venous catheters were first washed with $10 \mathrm{~mL}$ of normal saline solution then locked with $5 \mathrm{~mL}$ of the heparinized saline solution with concentration of $10 \mathrm{u} / \mathrm{mL}$. For arterial catheters, in the intervention group heparinized saline solution was used with concentration of $10 \mathrm{u} / \mathrm{mL}$ and in the control group $5 \mathrm{~mL}$ of normal saline was used. Both the patients and primary endpoint assessor were blind to composition of flushing solutions (double blinded trial).

\subsection{Statistical Analysis}

The data regarding 100 patients were analyzed by SPSS for windows statistical package v. 21.0 (SPSS inc., Chicago, IL, USA). Categorical variables were analyzed by Chisquare or Fisher's exact tests. Mean values of continuous variables were compared between the two study groups by independent sample t-test. P value $\leq 0.05$ was considered statistically significant. The approach and plan for statistical analysis was intention to treat analysis.

\section{Results}

Hundred patients were enrolled and completed the study based on the inclusion and exclusion criteria. Demographic and clinical data are presented in Table 1.

Sites of inserted Arterial catheters during three postoperative days follow up are presented in Tables 2 - 4 . The central venous catheters sites included: subclavian $17 \%$ and right internal jugular vein $83 \%$. In the current study, none of the central venous catheters were blocked.

Table 2 shows that manipulation and displacement of arterial catheters in the heparin group were $9(18 \%)$, versus those of the saline group 14 (28\%), which indicates no statistically significant difference. Arterial catheters lavage in the heparin group were $8(16 \%)$, versus those of the saline group $5(10 \%)$, which indicate no statistically significant difference (Pvalue $=0.745)$.

Table 1. Demographic and Clinical Data of the Patients a,b

\begin{tabular}{|c|c|c|c|c|c|}
\hline \multirow{2}{*}{$\begin{array}{l}\text { Variable } \\
\text { Age, } y\end{array}$} & \multicolumn{2}{|c|}{ Normal Saline $=\mathbf{5 0}$} & \multicolumn{2}{|c|}{ Heparinized Saline $=\mathbf{5 0}$} & \multirow{2}{*}{$\begin{array}{c}\text { PValue } \\
0.194\end{array}$} \\
\hline & $50(100)$ & $53.5 \pm 12.2$ & $50(100)$ & $49.7 \pm 11.2$ & \\
\hline Gender & & 0.418 & & 0.495 & \\
\hline Male & $39(78)$ & & $30(60)$ & & 0.052 \\
\hline Female & $11(22)$ & & $20(40)$ & & \\
\hline CABG Surgery & $33(66)$ & 0.479 & $27(54)$ & 0.503 & 0.221 \\
\hline Valve Surgery & $22(44)$ & 0.501 & $28(56)$ & 0.501 & 0.230 \\
\hline
\end{tabular}

a Abbreviation: CABG, Coronary Artery Bypass Graft.

b Data are presented as No. (\%) or Mean \pm SD or No.

Table 2. Table of Catheter-Related Information on the First Postoperative Day ${ }^{\mathrm{a}}$

\begin{tabular}{|c|c|c|c|}
\hline Variable & Normal Saline $=\mathbf{5 0}$ & Heparinized Saline $=\mathbf{5 0}$ & PValue \\
\hline Manipulation and displacement of arterial catheter & $14(28)$ & $9(18)$ & 0.492 \\
\hline Arterial catheter lavage ${ }^{b}$ & $5(10)$ & $8(16)$ & 0.745 \\
\hline Manipulation and displacement of venous catheter & $13(26)$ & $10(20)$ & 0.476 \\
\hline Venous catheter lavage ${ }^{b}$ & $9(18)$ & $6(12)$ & 0.401 \\
\hline Arterial catheter occlusion & $1(2)$ & $1(2)$ & 1.00 \\
\hline
\end{tabular}

a Data are presented as No.(\%).

b Catheter lavage: Need for repeated solution flush to maintain acceptable waveform. 
Ziyaeifard Met al.

Table 3. Table of Catheter-Related Information in the Second Postoperative Day

\begin{tabular}{lccc}
\hline Variable & Normal Saline $(\mathbf{n}=\mathbf{5 0})$ & Heparinized Saline $(\mathbf{n}=\mathbf{5 0})$ & P Value \\
\hline Manipulation and displacement of arterial catheter & $12(24)$ & $8(16)$ & 0.513 \\
Arterial catheter lavage $^{\text {a }}$ & $9(18)$ & $5(10)$ & 0.438 \\
Manipulation and displacement of venous catheter $^{\text {venous catheter lavage }}{ }^{\mathrm{a}}$ & $16(32)$ & $13(26)$ & 0.509 \\
Arterial catheter occlusion & $6(12)$ & $5(10)$ & 0.749 \\
\hline
\end{tabular}

${ }^{\mathrm{a}}$ Catheter lavage: Need for repeated solution flush to maintain acceptable waveform.

Table 4. Table of Catheter-Related Information on the Third Postoperative Day

\begin{tabular}{|c|c|c|c|}
\hline Variable & Normal Saline $(\mathbf{n}=\mathbf{5 0})$ & Heparinized Saline $(\mathbf{n}=\mathbf{5 0})$ & PValue \\
\hline Manipulation and displacement of arterial catheter & $17(34)$ & $13(26)$ & 0.676 \\
\hline Arterial catheter lavage $^{a}$ & $14(28)$ & $10(20)$ & 0.639 \\
\hline Manipulation and displacement of venous catheter & $17(34)$ & $14(28)$ & 0.517 \\
\hline venous catheter lavage ${ }^{a}$ & $5(10)$ & $8(16)$ & 0.372 \\
\hline Arterial catheter occlusion & $4(8)$ & $3(6)$ & 0.695 \\
\hline
\end{tabular}

${ }^{a}$ Catheter lavage: Need for repeated solution flush to maintain acceptable waveform.

Manipulation and displacement of venous catheters in the heparin group were 10 (20\%), versus those of the saline group 13 (26\%), which indicates no statistically significant difference. venous catheters lavage in the heparin group were 6 (12\%), versus the saline group $9(18 \%)$, which indicates no statistically significant difference $(\mathrm{P}$ value $=$ 0.401). Arterial catheters occlusion in the heparin group were $1(2 \%)$, versus the saline group $1(2 \%)$, which indicates no statistically significant difference $(P$ value $=1.0$ ).

Manipulation, displacement and lavage of arterial and venous catheters in the heparinized saline group and saline group were not significantly different in the 2nd postoperative day (Pvalues >0.05) (Table 3).

Manipulation and displacement of arterial catheters in the heparin group were not significantly different (P val$\mathrm{ue}=0.676$ ). Also, arterial catheters lavage in the heparin group were not significantly different $(P$ value $=0.639$ ). Manipulation and displacement of venous catheters in the heparin group were not significantly different (P val$\mathrm{ue}=0.517)$. Venous catheters lavage in the heparin group were not significantly different $(P$ value $=0.372$ ).

\section{Discussion}

The results of the current study showed no statistically significant difference regarding the patients' demographic characteristics such as age and gender between the two groups. The most common site of arterial catheters similar to those of the previous studies was radial artery. The proportion of arterial catheters placement sites including radial, brachial and femoral arteries were similar to those of the previous studies and there was no statistically significant difference between the two groups (16). The sites of venous catheters between the two groups were similar to those of the previous studies and not significantly different (8). In the current study, the arterial catheters manipulation including displacement and lavage were similar to those of the previous studies and there was no statistically significant difference between the two groups (5). Central venous catheters manipulation including displacement and lavage between the two groups of the current study was not significantly different. Arterial catheters occlusion between the two groups was not significantly different, similar to those of the previous studies. Also central venous catheters occlusions between the two groups were not significantly different, similar to those of the previous studies (16). Also the effect of heparinized saline with normal saline solutions in patency of peripheral venous catheters in the adult and newborn was studied, which were not significantly different $(14,15)$. In a current systematic review study Kordzadeh et al. (17) determined that, heparinized saline solution may be superior for long term use in arterial line. However the current study assessed this issue in short term period and found no significant difference. Cardiac surgery includes vascular and valve surgery, and in the current study there was no statistically significant difference between the two groups, but in the other studies were not surveyed. Variable alteration during three days of patients follow up including catheters occlusion and other parameters did not significantly change that was similar to the obtained results of the studies in which patients were followed up from several days to 12 months $(5,8,16,18)$.

Based on the finding of the current double blind randomized clinical trial, use of heparinized saline solutions compared with normal saline solutions did not prolong the patency of arterial and central venous catheters in short term postoperative period. Finding of the study suggested that normal saline solution can be used as the 
standard solution to prevent catheter occlusion after cardiac surgery. Therefore, all medical centers are suggested to prevent heparin complications such as allergic reaction, local tissue injury, bleeding, thrombus and thrombocytopenia related to use of heparin in patients with arterial and central venous catheter; normal saline solution can be used to prevent catheter occlusion as a safe alternative solution.

It seems that the use of heparinized saline and normal saline solution in preventing occlusion of arterial and central venous catheter has no difference. Our finding only applicable to cardiac surgery patients that have arterial or central venous line catheters for short time period ( 3 three days) post-operatively. Therefore these dada could not be generalizable to chronic medical situations.

\subsection{Limitations}

Arterial and venous catheters patency were assessed only for three postoperative days; therefore no data were provided on prolonged maintenance of indwelling catheters by heparinized or normal saline solutions flush. Regarding relatively low frequency of arterial or venous catheter occlusion, conducting large scale studies could assess the difference of heparinized and normal saline solutions flush better.

\section{Acknowledgements}

Authors wish to acknowledge their gratitude to the ethics committee of Tehran Rajaie cardiovascular medical and research center for granting approval to conduct this research. Authors would like to thanks all the colleagues in the intensive care unit and Mr. Mohammad Masbi and Mr. Mohammad Ali Najafikhah.

\section{Authors' Contributions}

Mohsen Ziyaeifard; conduct of study, administration technical and scientific revision of the article; Nahid Aghdaii: literature search and clinical analysis: Rasoul Azarfarin: data interpretation and clinical analysis: Azin Alizadehasl: data interpretation and critical revision of the article; Ghodrat Golbargian: data collection and manuscript preparation.

\section{References}

1. Cruzeiro PC, Camargos PA, Tatsuo ES, Picarro C, Campos BA, Paixao RM, et al. Percutaneous central venous catheterization through the external jugular vein in children: is inserting the guide wire into the superior vena cava essential for successful catheterization? J Pediatr Surg. 2012;47(9):1742-7.

2. Pittiruti M, Hamilton H, Biffi R, MacFie J, Pertkiewicz M, Espen. ESPEN Guidelines on Parenteral Nutrition: central venous catheters (access, care, diagnosis and therapy of complications). Clin Nutr. 2009;28(4):365-77.

3. Pinsky MR. Hemodynamic monitoring in the intensive care unit. Clin Chest Med. 2003;24(4):549-60.

4. Kim SH, Lilot M, Sidhu KS, Rinehart J, Yu Z, Canales C, et al. Accuracy and precision of continuous noninvasive arterial pressure monitoring compared with invasive arterial pressure: a systematic review and meta-analysis. Anesthesiology. 2014;120(5):1080-97.

5. Kulkarni M, Elsner C, Ouellet D, Zeldin R. Heparinized saline versus normal saline in maintaining patency of the radial artery catheter. Can J Surg. 1994;37(1):37-42.

6. Witkowski MC, Moraes MA, Firpo CM. Lack of difference between continuous versus intermittent heparin infusion on maintenance of intra-arterial catheter in postoperative pediatric surgery: a randomized controlled study. Rev Paul Pediatr. 2013;31(4):516-22.

7. Murphy GS, Marymont JH. Alternative anticoagulation management strategies for the patient with heparin-induced thrombocytopenia undergoing cardiac surgery.JCardiothorac Vasc Anesth. 2007;21(1):113-26.

8. Bertoglio S, Solari N, Meszaros P, Vassallo F, Bonvento M, Pastorino $\mathrm{S}$, et al. Efficacy of normal saline versus heparinized saline solution for locking catheters of totally implantable long-term central vascular access devices in adult cancer patients. Cancer Nurs. 2012;35(4):E35-42.

9. Fink MP, Abraham E, Vincent JL, Kochanek P. Textbook of critical care. Elsevier Saunders; 2008.

10. Halfman M, Reiner S. Quick Guide to Central Venous Access.Irvine, CA: Edwards Lifesciences LLC; 2002. pp.1-144.

11. Junqueira DR, Carvalho M, Perini E. Heparin-induced thrombocytopenia: a review of concepts regarding a dangerous adverse drug reaction. Rev Assoc Med Bras. 2013;59(2):161-6.

12. Napolitano LM, Warkentin TE, Almahameed A, Nasraway SA. Heparin-induced thrombocytopenia in the critical care setting: diagnosis and management. Crit Care Med. 2006;34(12):2898-911.

13. Robertson-Malt S, Malt GN, Farquhar V, Greer W. Heparin versus normal saline for patency of arterial lines. Cochrane Database Syst Rev. 2014;5:CD007364.

14. Sevrina RC. Effect Of Normal Saline Flush On Patency Of Peripheral Venous Catheters For The Prevention OfThrombophlebitis-A Randomized Control Trial. Belgaum, Karnataka: KLE University; 2013.

15. Arnts IJ, Heijnen JA, Wilbers HT, van der Wilt GJ, Groenewoud $J M$, Liem KD. Effectiveness of heparin solution versus normal saline in maintaining patency of intravenous locks in neonates: a double blind randomized controlled study. J Adv Nurs. 2011;67(12):2677-85.

16. Del Cotillo M, Grane N, Llavore M, Quintana S. Heparinized solution vs. saline solution in the maintenance of arterial catheters: a double blind randomized clinical trial. Intensive Care Med. 2008;34(2):339-43.

17. Kordzadeh A, Austin T, Panayiotopoulos Y. Efficacy of normal saline in the maintenance of the arterial lines in comparison to heparin flush: a comprehensive review of the literature.J Vasc Access. 2014;15(2):123-7.

18. Whitta RK, Hall KF, Bennetts TM, Welman L, Rawlins P. Comparison of normal or heparinised saline flushing on function of arterial lines. Crit Care Resusc. 2006;8(3):205-8. 